\title{
Outcomes of ovulation induction-intrauterine insemination in lean, overweight, and obese women with polycystic ovary syndrome
}

\author{
Michelle Krohn DO, ${ }^{1}$ Amy E.T. Sparks PhD, ${ }^{2}$ Divya K Shah MD ${ }^{2}$ \\ Keywords: Ovulation induction, intrauterine insemination, polycystic ovary syndrome, lean, overweight, \\ body mass index
}

\begin{abstract}
Objective: To compare pregnancy rates between lean, overweight, and obese women with polycystic ovary syndrome (PCOS) undergoing ovulation induction-intrauterine insemination (OI-IUI).
\end{abstract}

Design: Retrospective cohort study

Setting: Academic training program

Patient(s): All women with PCOS undergoing OI-IUI from January 2000 to November 2013.

Interventions: Ovulation induction-intrauterine insemination with oral (oral OI-IUI) or combined oral and injectable (combined OI-IUI) agents.

Main Outcome Measures: Clinical pregnancy rates (CPR) per IUI were compared in 198 women undergoing oral OI-IUI and combined OI-IUI. Multivariate logistic regression was used to calculate the odds of clinical pregnancy while adjusting for potential confounders. Results were expressed as adjusted odds ratios (OR) with 95\% confidence intervals (Cl), using lean women as the referent population.

Results: In lean PCOS patients undergoing combined OI-IUI, the unadjusted CPR was 52\%; CPR was comparatively lower in overweight
(22\%), obese (27\%) and morbidly obese (21\%) women for all cycles. Adjusting for age and duration of infertility, the odds of clinical pregnancy after combined OI-IUI was significantly diminished in overweight $(O R=0.27$ $\mathrm{Cl}=0.12-0.63)$, obese $(\mathrm{OR}=0.41, \mathrm{Cl}=0.20-0.83)$ and morbidly obese women $(\mathrm{OR}=0.33 \mathrm{Cl}=0.14$ 0.78 ) as compared to the lean PCOS referent. $A$ similar but non-significant trend was identified in women undergoing oral OI-IUI.

Conclusion: Lean women with PCOS have a higher CPR after combined OI-IUI as compared to their overweight, obese, and morbidly obese counterparts. Patients with a lean PCOS phenotype may preferentially benefit from this treatment approach.

${ }^{1}$ University of lowa Hospitals and Clinics, Department of Obstetrics and Gynecology, lowa City, lowa

${ }^{2}$ University of lowa Hospitals and Clinics, Department of Obstetrics and Gynecology, Division of Reproductive Endocrinology and Infertility, lowa City, lowa

\section{Introduction}

The prevalence of polycystic ovary syndrome (PCOS) in the general female population ranges from $5-10 \%,{ }^{1,2}$ and

Please cite this paper as: Krohn M, Sparks AET, Shah DK. Outcomes of ovulation induction-intrauterine insemination in lean, overweight, and obese women with polycystic ovary syndrome. Proceedings in Obstetrics and Gynecology, 2015;5(2):Article 5 [ 9 p.]. Available from: http://ir.uiowa.edu/pog/ Free full text article.

Corresponding author: Michelle Krohn, DO, Department of Obstetrics and Gynecology, University of lowa Hospitals and Clinics, 200 Hawkins Drive, lowa City, IA 52242. Phone: 319-356-4484, michelle-krohn@uiowa.edu

Financial Disclosure: The authors report no conflict of interest.

Copyright: (c) 2015 Krohn et al. This is an open-access article distributed under the terms of the Creative Commons Attribution License, which permits unrestricted use, distribution, and reproduction in any medium, provided the original author and source are credited. 
the disease accounts for nearly $73 \%$ of cases of anovulatory infertility. ${ }^{3,4}$ The disease process is characterized by variable degrees of hyperandrogenism, morphologic changes in the ovary, inappropriate gonadotropin secretion, and insulin resistance - all of which contribute to the phenotype of ovulatory dysfunction and infertility. ${ }^{5}$

Obesity, defined by the World Health Organization (WHO) as a body mass index (BMI) of $>30 \mathrm{~kg} / \mathrm{m}^{2}$, is an increasing health concern in the United States. Recent estimates identify greater than $31 \%$ of reproductive-aged American women as obese, and nearly $55 \%$ as overweight $\left(\mathrm{BMI}>25 \mathrm{~kg} / \mathrm{m}^{2}\right) .{ }^{6} \mathrm{~A}$ majority of PCOS patients are overweight or obese, ${ }^{1}$ and obesity contributes to several metabolic alterations that interfere with normal ovulation through increased peripheral aromatization resulting in hyperestrogenemia, decreased sexhormone binding globulin (SHBG) resulting in increased free testosterone levels, and hyperinsulinemia resulting in higher thecal androgen production. ${ }^{4,7}$ Despite its strong association with the disease, obesity is not requisite for the diagnosis of PCOS and approximately $25-30 \%$ of women with PCOS are of normal BMI $\left(18.5-25 \mathrm{~kg} / \mathrm{m}^{2}\right)$. These women are thought to be at lower risk for the metabolic dysfunction typically associated with PCOS, indicating that obesity, rather than PCOS, may be the primary contributor to this risk. ${ }^{8}$

There is little data describing fertility potential in the lean PCOS variant. A few recent studies have compared in vitro fertilization (IVF) outcomes in the lean versus obese PCOS populations, with two groups reporting lower pregnancy rates in obese PCOS patients $^{9,10}$ and a third reporting no difference. ${ }^{11}$ Even less is known about the impact of BMI on pregnancy outcome in PCOS patients undergoing ovulation induction (OI), despite this being considered the first line treatment for this population. We sought to compare clinical pregnancy rates (CPR) between lean, overweight, and obese PCOS patients undergoing ovulation induction and intrauterine insemination (OI-IUI) with oral (oral OI-IUI) or combined oral and injectable medications (combined OI-IUI).

\section{Materials and Methods}

\section{Study Population}

The study population included women aged 21-43 who underwent OI-IUI at the University of lowa between January 2000 and November 2013. Analyses were restricted to women with the sole infertility diagnosis of PCOS as defined by the 2003 Rotterdam criteria. ${ }^{12}$ Couples who had multiple infertility diagnoses (including endometriosis, tubal factor, or diminished ovarian reserve) were excluded, as were those with severe male factor, as defined by a pre-wash total motile sperm count of less than 10 million averaged over all semen analyses performed. Pregnancy outcome data was lacking for three patients during the study period and these individuals were also excluded from analyses. The method of ovulation induction was selected by the individual provider, and consisted of either oral ovulation induction with clomiphene citrate or letrozole, or combined oral/injectable ovulation induction with clomiphene citrate or letrozole in conjunction with gonadotropins. Oral ovulation induction cycles were typically monitored using home urinary luteinizing 
hormone (LH) kits, whereas combined oral/injectable ovulation induction cycles were monitored with serial sonographic measurements of follicular growth beginning on treatment day 8 . Spontaneous ovulation was permitted in oral ovulation induction cycles, whereas $250 \mathrm{mcg}$ of subcutaneous recombinant human chorionic gonadotropin (hCG) was used to trigger ovulation in combined oral/injectable ovulation induction cycles. IUI was performed 36 hours after hCG trigger or 24 hours after a positive home urinary LH kit. The study was approved by the Institutional Review Board at The University of lowa.

\section{Exposure and outcome variables}

Body mass index was calculated as weight in kilograms over height in meters squared based on measurements obtained at the initiation of treatment. Body mass index was categorized based on World Health Organization cut points as lean $(<25$ $\left.\mathrm{kg} / \mathrm{m}^{2}\right)$, overweight $\left(25.0-29.9 \mathrm{~kg} / \mathrm{m}^{2}\right)$, obese $\left(30.0-39.9 \mathrm{~kg} / \mathrm{m}^{2}\right)$, or morbidly obese $\left(>40 \mathrm{~kg} / \mathrm{m}^{2}\right)$. Lean women were used as the referent population for all comparisons. The primary outcome was clinical pregnancy, defined as sonographic evidence of intrauterine gestational sac on ultrasound. Clinical pregnancy rates were calculated per completed IUI.

\section{Potential confounding variables}

Female age, parity, and IUI attempt number were documented at cycle start. Other patient variables assessed at the time of IUI included current smoking status (yes/no), current metformin use (yes/no), semen parameters (average pre-wash total motile count averaged for all IUI cycles), and duration of infertility (months). Documented cycle characteristics included type of OI (letrozole, clomiphene citrate, +/gonadotropins), utilization of an hCG trigger (yes/no), and for combined OI-IUI cycles, total number of follicles and number of follicles $>15 \mathrm{~mm}$ on day of hCG trigger.

\section{Statistical Analysis}

Logistic regression was used to estimate the effect of BMI on clinical pregnancy, adjusting for potential confounders including parity, cycle number, smoking status, metformin use, semen parameters, type of oral agent used, utilization of hCG trigger, and total number of follicles and number of follicles $>15 \mathrm{~mm}$ on day of hCG trigger for combined OI-IUI cycles. Female age and duration of infertility were included a priori as covariates in the model. Other potential confounders were examined but resulted in a less than $10 \%$ change in the effect estimates and were therefore not included in the final model. Generalized estimating equations were used to account for the correlation between multiple cycles in the same patient. Results were expressed as adjusted odds ratios (OR) with 95\% confidence intervals $(\mathrm{Cl})$, using lean women as the referent population.

\section{Results}

One hundred ninety eight women with PCOS contributed a total of 440 cycles to the analysis: 114 cycles from 48 lean women, 87 cycles from 38 overweight women, 146 cycles from 65 obese women, and 93 cycles from 47 morbidly obese women. Demographic characteristics of the study population are listed in Table 1. Fifty-seven percent of participants were obese or 
morbidly obese. Patient age and duration of infertility both rose with increasing BMI, whereas smoking status, parity, and average total motile sperm counts were similarly distributed across all BMI categories. Use of metformin was more common among obese women. There was more clomiphene use in the oral OI-IUI cycles (66\% of all cycles), and more letrozole use $(75 \%$ of all cycles) in the combined OI-IUI cycles. The total number of follicles measured on ultrasound at time of hCG trigger in combined OI-IUI cycles was higher for lean patients than overweight or obese patients, though the median number of follicles $>15 \mathrm{~mm}$ at time of hCG injection did not substantially differ between BMI categories.

Table 1. Characteristics of 198 women and 440 ovulation induction and intrauterine insemination cycles among women with polycystic ovary syndrome

\begin{tabular}{|c|c|c|c|c|}
\hline & $\begin{array}{c}\text { Lean } \\
(\mathrm{BMI}<25)\end{array}$ & $\begin{array}{l}\text { Overweight } \\
\text { (BMI 25-29.9) }\end{array}$ & $\begin{array}{c}\text { Obese } \\
\text { (BMI 30-39.9) }\end{array}$ & $\begin{array}{c}\text { Morbidly obese } \\
\text { (BMI } \leq \mathbf{4 0 . 0 )}\end{array}$ \\
\hline Patient characteristics & $\mathrm{N}=48$ & $\mathrm{~N}=38$ & $\mathrm{~N}=65$ & $\mathrm{~N}=47$ \\
\hline Age at insemination (years) ${ }^{a}$ & $29.2(3.8)$ & $28.7(4.1)$ & $30.4(4.3)$ & $31.1(4.6)$ \\
\hline $\begin{array}{l}\text { Parity }^{\mathrm{b}} \\
0 \\
1 \\
2\end{array}$ & $\begin{array}{c}42(89 \%) \\
3(6 \%) \\
2(4 \%)\end{array}$ & $\begin{array}{c}30(81 \%) \\
6(16 \%) \\
1(3 \%)\end{array}$ & $\begin{array}{c}54(83 \%) \\
9(14 \%) \\
2(3 \%)\end{array}$ & $\begin{array}{c}39(83 \%) \\
7(15 \%) \\
1(2 \%)\end{array}$ \\
\hline Current smoker $^{\mathrm{b}}$ & $4(9 \%)$ & $2(7 \%)$ & $11(20 \%)$ & $6(18 \%)$ \\
\hline Duration of infertility (months) $^{c}$ & $18(12-24)$ & $24(18-33)$ & $27(18-48)$ & $36(18-60)$ \\
\hline Cycle characteristics & $\mathrm{N}=114$ & $\mathrm{~N}=87$ & $\mathrm{~N}=146$ & $\mathrm{~N}=93$ \\
\hline $\begin{array}{l}\text { Oral OI agent } \\
\text { Clomiphene } \\
\text { Letrozole }\end{array}$ & $\begin{array}{l}50(74 \%) \\
18(26 \%) \\
\end{array}$ & $\begin{array}{l}18(44 \%) \\
23(56 \%)\end{array}$ & $\begin{array}{l}41(80 \%) \\
10(20 \%)\end{array}$ & $\begin{array}{l}20(57 \%) \\
15(43 \%)\end{array}$ \\
\hline $\begin{array}{l}\text { Combined OI agents } \\
\text { Clomiphene } \\
\text { Letrozole }\end{array}$ & $\begin{array}{c}9(20 \%) \\
37(80 \%)\end{array}$ & $\begin{array}{l}15(33 \%) \\
31(67 \%)\end{array}$ & $\begin{array}{l}26(27 \%) \\
69(73 \%)\end{array}$ & $\begin{array}{l}11(19 \%) \\
47(81 \%)\end{array}$ \\
\hline hCG trigger ${ }^{b}$ & $59(52 \%)$ & $51(59 \%)$ & $102(70 \%)$ & $66(72 \%)$ \\
\hline Metformin ${ }^{b}$ & $13(11 \%)$ & $15(17 \%)$ & $50(34 \%)$ & $27(29 \%)$ \\
\hline Average total motile sperm count $\left(\mathrm{x}^{10^{6}}\right)^{\mathrm{C}}$ & $64.5(33-128)$ & $57(33-153)$ & $71.5(40-134)$ & $57(30-100)$ \\
\hline Combined OI-IUI cycles only & $\mathrm{N}=46$ & $\mathrm{~N}=44$ & $\mathrm{~N}=90$ & $\mathrm{~N}=52$ \\
\hline Total follicles $^{\mathrm{c}}$ & $6(4-28)$ & $4(3-6)$ & $4(2-16)$ & $3(2-6)$ \\
\hline Follicles $>15 \mathrm{~mm}^{\mathrm{c}}$ & $1(1-3)$ & $2(1-2)$ & $1(1-2)$ & $1(1-2)$ \\
\hline
\end{tabular}

Note: $\mathrm{BMI}=$ body mass index; $\mathrm{OI}=$ ovulation induction; $\mathrm{hCG}=$ human chorionic gonadotropin; IUI= intrauterine insemination

aResults expressed as mean (SD)

bResults expressed as n (\%)

cResults expressed as median (interquartile range)

Unadjusted rates of clinical pregnancy after the first cycle of oral OI-IUI showed no consistent association with patient $\mathrm{BMI}$, but trended downward when all cycles were examined (Table 2).
Adjusted analyses did not reveal a significant difference in odds of clinical pregnancy in overweight and obese women as compared to the lean PCOS referent population (Figure 1). 
Table 2. Unadjusted rates of clinical pregnancy in $\mathbf{4 4 0}$ ovulation induction and intrauterine insemination cycles among women with polycystic ovary syndrome

\begin{tabular}{|c|c|c|c|c|}
\hline & $\begin{array}{c}\text { Lean } \\
(\mathrm{BMI}<25)\end{array}$ & $\begin{array}{l}\text { Overweight } \\
\text { (BMI 25-29.9) }\end{array}$ & $\begin{array}{c}\text { Obese } \\
\text { (BMI 30-39.9) }\end{array}$ & $\begin{array}{l}\text { Morbidly obese } \\
\text { (BMI } \leq 40.0)\end{array}$ \\
\hline ORAL OI-IUI & $\mathrm{N}=27 / 68$ & $\mathrm{~N}=19 / 41$ & $\mathrm{~N}=22 / 51$ & $\mathrm{~N}=18 / 35$ \\
\hline \multicolumn{5}{|l|}{ Clinical pregnancy rate ${ }^{a}$} \\
\hline $1^{\text {st }}$ cycle & $4(15 \%)$ & $4(21 \%)$ & $2(9 \%)$ & $3(17 \%)$ \\
\hline All cycles & $14(21 \%)$ & $6(15 \%)$ & $8(16 \%)$ & $4(11 \%)$ \\
\hline COMBINED OI-IUI & $N=21 / 46$ & $N=19 / 46$ & $\mathrm{~N}=43 / 95$ & $N=29 / 58$ \\
\hline \multicolumn{5}{|l|}{ Clinical pregnancy rate ${ }^{a}$} \\
\hline $1^{\text {st }}$ cycle & $13(62 \%)$ & $1(5 \%)$ & 15 (35\%) & 5 (17\%) \\
\hline All cycles & $24(52 \%)$ & $10(22 \%)$ & $26(27 \%)$ & $12(21 \%)$ \\
\hline
\end{tabular}

Note: $\mathrm{BMI}=$ body mass index; $\mathrm{OI}=$ ovulation induction; IUI=intrauterine insemination; $\mathrm{N}=$ number of first intrauterine insemination cycles / total number of intrauterine insemination cycles

aResults expressed as n (\%)

In lean PCOS patients undergoing combined OI-IUI, the unadjusted CPR for all cycles was 52\%; CPR was comparatively lower in overweight (22\%), obese (27\%) and morbidly obese (21\%) women (Table 2). Adjusting for age and duration of infertility, the odds of clinical pregnancy was significantly diminished in overweight, obese, and morbidly obese women with PCOS undergoing combined OI-IUI as compared to the lean referent (Figure 2)

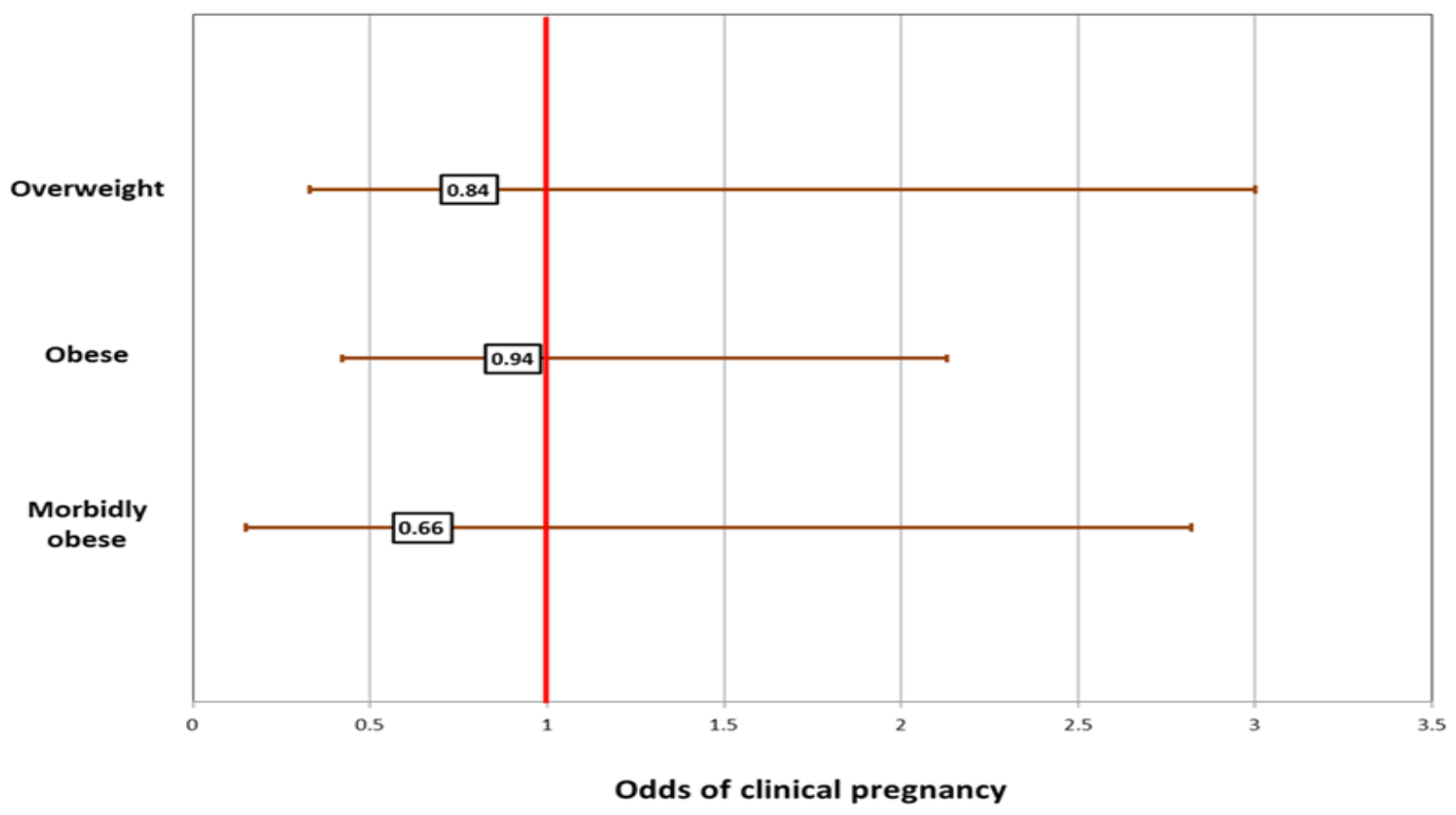

Figure 1. Adjusted odds of clinical pregnancy after oral ovulation induction and intrauterine insemination in women with polycystic ovary syndrome 


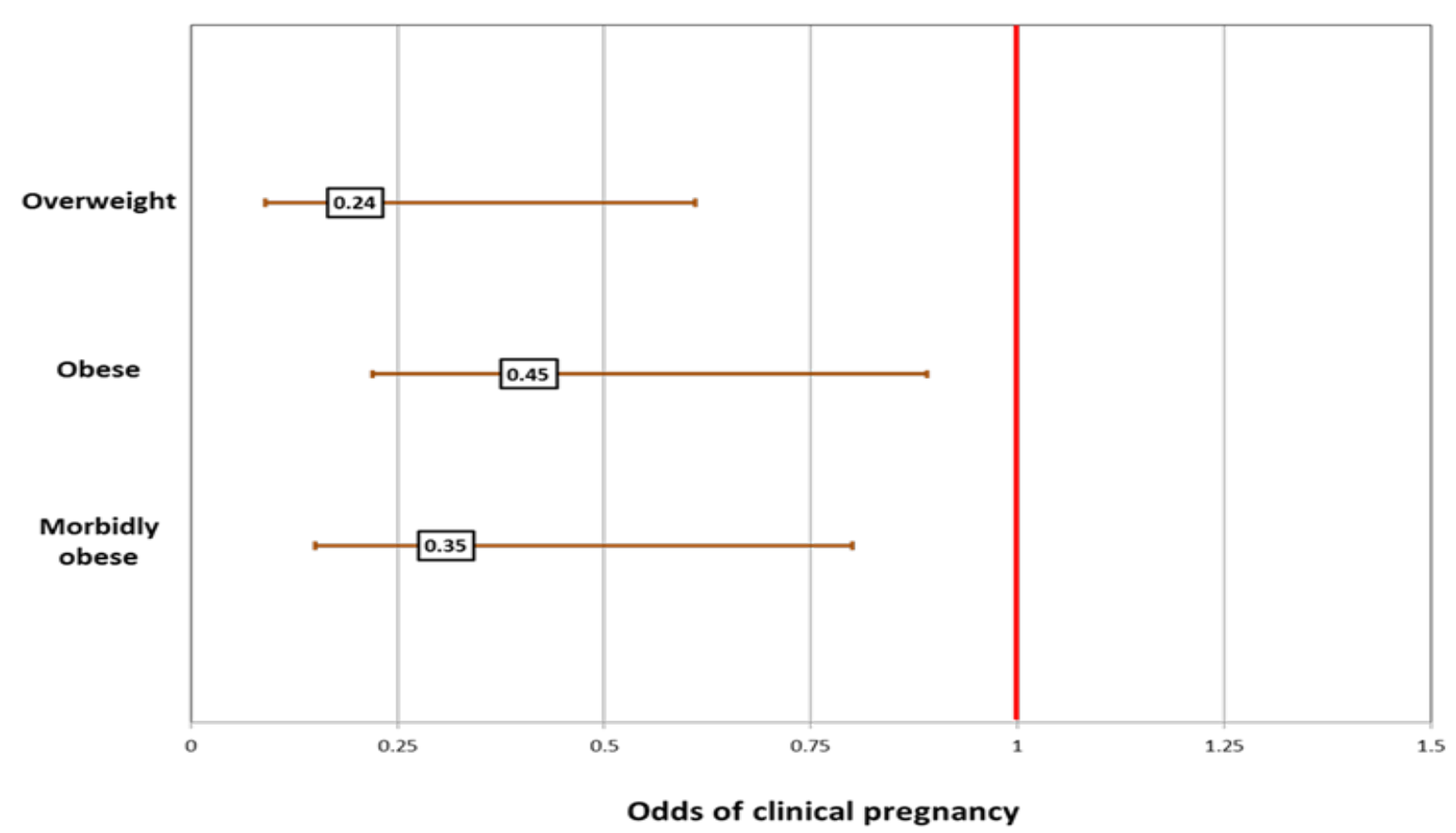

Figure 2. Adjusted odds of clinical pregnancy after combined oral and injectable ovulation induction and intrauterine insemination in women with polycystic ovary syndrome

\section{Discussion}

The results of this study demonstrate a significant negative association between increasing patient $\mathrm{BMI}$ and rates of clinical pregnancy in women with PCOS undergoing combined ovulation induction and intrauterine insemination with oral and injectable medications. Although a similar association was not identified in women with PCOS undergoing ovulation induction and intrauterine insemination with oral agents, analyses were limited by the small number of total pregnancies in this population and the possibility of a difference cannot therefore be excluded.
Retrospective studies have evaluated the effects of BMI on IVF cycle outcomes in women with PCOS, ${ }^{9-11}$ with discrepant results. A recent study demonstrated a $66 \%$ lower odds of clinical pregnancy in obese (BMl>30 $\mathrm{kg} / \mathrm{m}^{2}$ ) as compared to lean (BMI<25 $\mathrm{kg} / \mathrm{m}^{2}$ ) women with PCOS after adjusting for female age, gravidity, day 3 follicle stimulating hormone (FSH), and male factor infertility. ${ }^{10}$ A similar increase in clinical pregnancy rates in lean women with PCOS as compared to those with $\mathrm{BMI}>25 \mathrm{~kg} / \mathrm{m}^{2}$ was identified by a second study that also demonstrated a preferential benefit of $\mathrm{GnRH}$-agonist stimulation protocols in 
the lean cohort. $^{9}$ A third study comparing lean and obese women without PCOS to lean and obese women with PCOS demonstrated a greater number of retrieved oocytes in lean as compared to obese women with PCOS, but noted comparable rates of clinical pregnancy and live birth in all groups. The results of this study were significantly limited by its small sample size which included only six lean patients with PCOS. ${ }^{11}$ These studies suggest that lean PCOS patients may have a differential response to ovarian stimulation in the setting of IVF, but could not comment on the association with more conservative types of infertility treatment.

Our data appear consistent with the few existing studies examining the association between patient BMI and outcome of ovulation induction in women with PCOS. The multi-center, randomized control trial comparing clomiphene and metformin for ovulation induction in women with PCOS by Legro and colleagues demonstrated significantly higher live birth rates in subjects with $\mathrm{BMI}<30 \mathrm{~kg} / \mathrm{m}^{2}{ }^{5}$ A posthoc analysis of the data demonstrated a negative association between increasing patient BMI and rates of clinical pregnancy similar to that identified in our study. After ovulation induction with clomiphene, CPR ranged from $36.8 \%$ in women with $\mathrm{BMI}<30 \mathrm{~kg} / \mathrm{m}^{2}$ to $19.0 \%$ in women with BMI $30-34 \mathrm{~kg} / \mathrm{m}^{2}$ and $19.1 \%$ in women with $\mathrm{BMI}>35 \mathrm{~kg} / \mathrm{m}^{2}$.

A single retrospective study of 270 women directly investigated the impact of obesity on cycle outcome in women with PCOS undergoing ovulation induction. Treatment was initiated with clomiphene, and if no ovulation was documented in three cycles despite dose escalation, extended to gonadotropins. Women who ovulated successfully with clomiphene were given up to six cycles to conceive before gonadotropin therapy. The authors identified lower rates of ovulation and clinical pregnancy with increasing BMI, but data were unadjusted and did not account for key confounding factors including patient age. ${ }^{4}$ The study demographics also indicated a mean BMI of $28.5+/-5.5 \mathrm{~kg} / \mathrm{m}^{2}$, which may limit the generalizability of its results to populations with a higher prevalence of obesity.

Some key limitations should be considered when interpreting our results. The study population was identified retrospectively from our institution's database of couples undergoing IUI. Pregnancy rates could accordingly only be determined per IUI, not per cycle start. Cycles that were cancelled prior to IUI were therefore not included in the database, which may falsely elevate the overall rates of clinical pregnancy. This may have particular relevance in lean women with PCOS undergoing combined OI-IUI, a population with a significant risk of cycle cancellation due to multi-follicular development, where our unadjusted pregnancy rates were as high as 5060\%. A false elevation in CPR among lean women could overestimate the potential negative impact of obesity on odds of clinical pregnancy in the PCOS population, thus skewing our results away from the null hypothesis. This is supported by the absence of an association in the oral ovulation induction group where cycle cancellation rates are likely to be low. It can be argued, however, that morbidly obese women may also have a high risk of 
cycle cancellation due to provider desire to avoid multiple gestation pregnancies due to the significant obstetric consequences in this population. If this risk outweighs that of lean women, this may underestimate the impact of obesity on cycle outcome and skew data towards the null. It is worth noting, however, that adjusting for the total number of follicles and number of follicles $>15 \mathrm{~mm}$ on the day of hCG trigger did not substantially change the effect estimates, suggesting that the negative impact of obesity on odds of clinical pregnancy may be independent of differences in ovarian response to OI. Additional limitations include the nonstandardized selection of patients for either oral or combined OI-IUI as well as the lack of statistical power to examine other outcomes of interest such as miscarriage or multiple pregnancy.

Despite these limitations, this study is unique in its ability to examine the association between a wide range of patient BMI and outcome of ovulation induction in a well-defined cohort of PCOS patients. The large number of total IUI cycles afforded us the ability to account for variables including age and duration of infertility that may confound the relationship between $\mathrm{BMI}$ and cycle outcome, particularly in the combined OI-IUI cycles. Future prospective validation of these findings would afford the ability to examine differences in the medication dose required to achieve ovulation and account for the impact of cycle cancellation.

Given the limited existing literature on response to ovulation induction in these populations, this study provides useful data for patient counseling with respect to infertility treatment selection. The results of this study suggest that lean women with PCOS have higher pregnancy rates after combined OI-IUI with oral and injectable agents as compared to their overweight, obese, and morbidly obese counterparts, and may preferentially benefit from this treatment approach.

\section{References}

1. Moran C, Arriaga M, Rodriguez G, Moran S. Obesity differentially affects phenotypes of polycystic ovary syndrome. Int J Endocrinol. 2012;2012:317241. doi: 10.1155/2012/317241. Epub 2012 Jul 8. PubMed PMID: 22829818.

2. Caglar GS, Kahyaoglu I, Pabuccu R, Demirtas S, Seker R. Anti-Mullerian hormone and insulin resistance in classic phenotype lean PCOS. Arch Gynecol Obstet. 2013 Oct;288(4):90510. doi: 10.1007/s00404-013-2833-9. Epub 2013 Apr 4. PubMed PMID: 23553200.

3. Beydoun HA, Stadtmauer L, Beydoun MA, Russell H, Zhao $Y$, Oehninger S. Polycystic ovary syndrome, body mass index and outcomes of assisted reproductive technologies. Reprod Biomed Online. 2009 Jun;18(6):856-63. http://dx.doi.org/10.1016/S14726483(10)60037-5 PubMed PMID: 19490792.

4. Al-Azemi M, Omu FE, Omu AE. The effect of obesity on the outcome of infertility management in women with polycystic ovary syndrome. Arch Gynecol Obstet. 2004 Dec;270(4):20510. Epub 2003 Aug 29. PubMed PMID: 12955532. 
5. Legro RS, Barnhart HX, Schlaff WD, Carr BR, Diamond MP, Carson SA, Steinkampf MP, Coutifaris C, McGovern PG, Cataldo NA, Gosman GG, Nestler JE, Giudice LC, Leppert PC, Myers ER; Cooperative Multicenter Reproductive Medicine Network. Clomiphene, metformin, or both for infertility in the polycystic ovary syndrome. N Engl J Med. 2007 Feb 8;356(6):551-66. http://dx.doi.org/10.1056/NEJMoa06397 1 PubMed PMID: 17287476.

6. Flegal KM, Carroll MD, Kit BK, Ogden $\mathrm{CL}$. Prevalence of obesity and trends in the distribution of body mass index among US adults, 1999-2010. JAMA. 2012 Feb 1;307(5):491-7. doi: 10.1001/jama.2012.39. Epub 2012 Jan 17. PubMed PMID: 22253363.

7. Fritz MA, Speroff L. Clinical gynecologic endocrinology and infertility. 8th ed. Philadelphia : Wolters Kluwer Health/Lippincott Williams \& Wilkins; 2011.

8. Dokras A, Jagasia $D H$, Maifeld $M$, Sinkey CA, VanVoorhis BJ, Haynes WG. Obesity and insulin resistance but not hyperandrogenism mediates vascular dysfunction in women with polycystic ovary syndrome. Fertil Steril. 2006 Dec;86(6):1702-9. Epub 2006 Oct 24.

http://dx.doi.org/10.1016/j.fertnstert.200 6.05.038 PubMed PMID: 17067587.

9. Orvieto $\mathrm{R}$, Nahum $\mathrm{R}$, Meltcer $\mathrm{S}$, Homburg R, Rabinson J, Anteby EY, Ashkenazi J. Ovarian stimulation in polycystic ovary syndrome patients: the role of body mass index. Reprod Biomed Online. 2009 Mar;18(3):333-6. http://dx.doi.org/10.1016/S14726483(10)60090-9 PubMed PMID: 19298731.
10. Bailey AP, Hawkins LK, Missmer SA, Correia KF, Yanushpolsky EH. Effect of body mass index on in vitro fertilization outcomes in women with polycystic ovary syndrome. Am J Obstet Gynecol. 2014 Aug;211(2):163.e1-6. doi: 10.1016/j.ajog.2014.03.035. Epub 2014 Mar 18. PubMed PMID: 24657792.

11. McCormick B, Thomas M, Maxwell R, Williams D, Aubuchon M. Effects of polycystic ovarian syndrome on in vitro fertilization-embryo transfer outcomes are influenced by body mass index. Fertil Steril. 2008 Dec;90(6):2304-9. doi: 10.1016/j.fertnstert.2007.10.077. Epub 2008 Jan 14. PubMed PMID: 18191852.

12. Rotterdam ESHRE/ASRM-Sponsored PCOS Consensus Workshop Group. Revised 2003 consensus on diagnostic criteria and long-term health risks related to polycystic ovary syndrome. Fertil Steril. 2004 Jan;81(1):19-25. http://dx.doi.org/10.1016/j.fertnstert.200 3.10.004 PubMed PMID: 14711538. 